UCRL-JC-113874

PREPRINT

\title{
Automated Assistance for Detecting Malicious Code
}

\author{
R. Crawford, P. Kerchen, K. Levitt, \\ R. Olsson, M. Archer, M. Casillas
}

This paper was prepared for submittal to the Sixth International Computer Security \& Virus Conference \& Expo

March 10-12, 1993

New York, New York

June 18, 1993

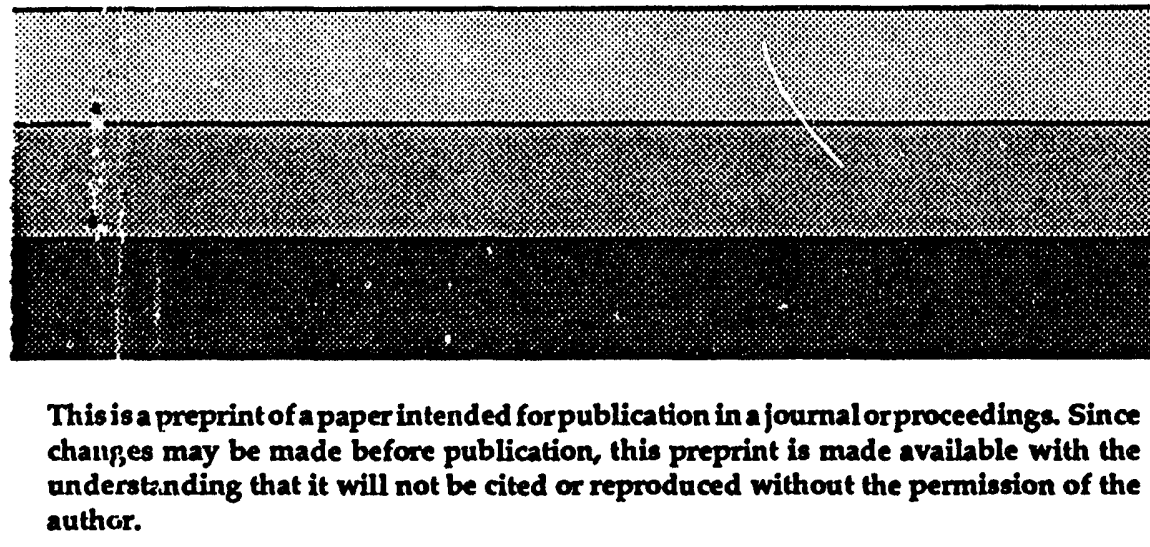

\section{MASTER}

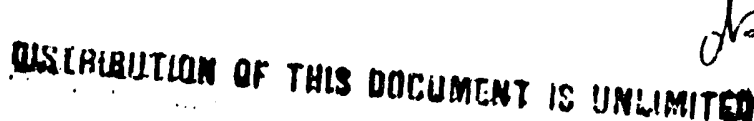




\section{DISCLAIMER}

This document was prepared as an account of work sponsored by an agency of the United States Government. Neither the United States Government nor the the United States Government. Neither the employees, makes any warranty, express or implied, or assumes any legal liability or responsibility for the accuracy, or impled, or usefulness of any information, apparatus, product, or process completeness, or usefulness of any information, apparatus, product, or process Reference herein to any specific commercial product, process, or service by trade Reference herein to any specturer, or otherwise, does not necessarily constitute or imply its endorsement, recommendation, or favoring by the United States Government or the University of California. The views and opinions of uthors expressed herein do not necessarily state or reflect those of the Unitea States Government or the University of California, and shall not be used for advertising or product endorsement purposes. 


\title{
Automated Assistance for Detecting Malicious Code *
}

\author{
R. Crawford, P. Kerchen, K. Levitt, R. Olsson, M. Archer, M. Casillas \\ Department of Computer Science \\ University of California, Davis \\ Davis, CA 95616 \\ Email: virus@cs.ucdavis.edu
}

\begin{abstract}
This paper gives an update on our continuing work on the Malicious Code Testbed (MCT). The MCT is a semi-automated tool, operating in a simulated, cleanroom environment, that is capable of detecting many types of malicious code, such as viruses, Trojan horses, and time/logic bombs. The MCT allows security analysts to check a program before installation, thereby avoiding any damage a nalicious program might inflict.
\end{abstract}

Keywords: Detection of Malicious Code, Static Analysis, Dynamic Analysis.

\section{Introduction}

The Malicious Code Testbed (MCT) was originally designed to use both static and dynamic analysis tools developed at the University of California, Davis, that have been shown to be effective against certain types of malicious code. One goal of the testbed is to enhance the power of similar tools by using them in a complementary fashion to detect more general cases of malicious code.

In our report to this conference last year [1], we presented a design overview of the MCT. In the present paper, we report on our progress towards upgiading the MCT environment for dynamic analysis.

Although, in principle, the notion of a Malicious Code Testbed is independent of any particular operating system or architectural platform, our initial implementation efforts have focused on simulating a DOS operating system running on PC architectures. This design decision was made primarily because the PC/DOS elivironment is so widespread and accessible to intrusions; thus this environment is the one that has engendered the most real-world malicious code we can use to challenge our detection techniques.

Sections 2 and 3 provide background material on malicious code and current detection methods. Section 4 reviews the use of events in dynamic aralysis techniques, and Section 5 describes the architecture of the MCT. Section 6 presents some results from our experience using the MCT on malicious code.

"SPONSORS: Lawrence Livermore National Laboratory, U.S. Department of Energy

Work performed under the auspices of the U.S. Department of Energy by Lawrence Livermore National Laboratory under Contract W-7405-Eng-48.

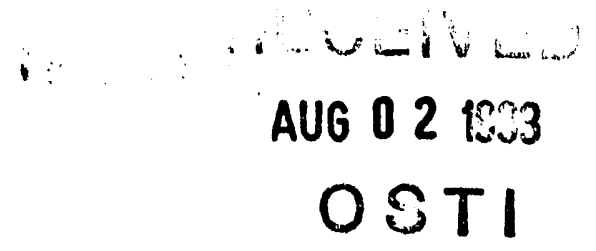




\section{Malicious Code - A Brief Overview}

In recent years, various forms of malicious code have appeared on virtually all major families of computer platform. The prevalence of malicious code - Trojan horses, time bombs, worms, and viruses - threatens the traditional "open systems" approach that has evolved in the academic realm, as well as in much of the commercial sector.

The current situation in the personal computer arena may be indicative of future trends in workstation and mainframe environments. On PC systems - where literally hundreds of computer viruses, time bombs, and Trojan horses have proliferated the problem is caused by rogue programs that unwittingly are invited in to the system. Thus malicious code may be inserted into almost any type of computer system via these same avenues - "shareware" may be installed, or malicious code might be produced in-house by a disgruntled employee, or a program containing malicious code might even be purchased from a legitimate vendor of commercial software.

Our definition of what constitutes "malicious" code shall address only the probable effects of executing such code; we shall not concern ourselves with the "original intent" of the (possibly unknown) writer. Although the intentions of the writer may be crucial in determining legal culpability - e.g., whether malice and forethought were present to include such considerations within the scope of our "working definition" for malicious code would clearly render the problem incomputable.

Yet even using our restricted, operational definition of "malicious code", the problem of malicious code detection - in the most general case - is not decidable by purely formal methods. This follows not merely from the results of [4] [2] [3], but sather because the inherent semantics of the problem statement demand that a value judgement regarding the nature of the code's probable effects be rendered. But because doing so would require that the intent of the program's potential users be considered, no article of faith akin to Church's Thesis can serve to bridge the gap between our intuitive sense of "malicious effects", and algorithmic solutions. It would seem that, in all but the most severely restricted programming environments, the problem statement must remain a fuzzy one.

Thus, although no algorithm that identifies malicious code in all environments and in all guises can exist, a number of techniques already exist for coping with certain restricted forms of malicious code. Since the problem cannot with certainty be prevented in current programming environments, it must be managed instead.

This idea forms the basis of the Malicious Code Testbed - an automated assistant whose mission it is to perform the "grunt work" necessary to aid a human analyst in detecting not only currently known forms of malicious code, but also mutated or entirely novel forms. Given the absence of a decision procedure for malicious code, such a testbed would allow one to examine a program to ascertain whether or not it is suspicious.

We first discuss the most prevalent methods of coping with malicious code, and then describe some of our previous work aimed at providing defenses against malicious code. Then we explore in greater detail the Malicious Code Testbed. 


\section{A Sample of Current Methods for Coping with Malicious Code}

Presently, the majority of malicious code defenses are concerned with computer viruses. However, some are more broadly applicable to malicious code in general. These methods may be divided into two distinct classes depending on when they are applied: as a pre-execution check or at run time. Pre-execution techniques are applied to a suspicious program before it can be executed by a user. In contrast, run time methods are actually applied to the program as it executes, in hopes of stopping the program before it can cause damage or allow a virus to propagate. Another taxonomy of malicious code defenses divides all methods into the categories of static or dynamic analysis. Although most static analysis techniques are applied as pre-execution checks, certain static analysis techniques can be applied at run time. Similarly, although most dynamic analysis techniques are applied as run time checks, certain dynamic analysis techniques (such as our own Malicious Code Testbed) can be applied as pre-execution checks.

Many of the more sophisticated pre-execution methods rely on the prior existence of a copy of the program that is assumed to be "clean", perhaps because it was originally written by a trusted programmer and then translated into an executable file by a trusted compiler on a secure system. One such method computes cryptographic checksums that are characteristic of that trusted executable file, and embeds them in that file. [6] The file is then copied to an insecure environment, whose operating system will not allow a user to execute any program until it has recomputed what those checksums should be and compared those values with the ones actually embedded in the program. In this way, most alterations made to a trusted executable file after it leaves the secure system can be detected before the program is executed in the insecure environment.

It is important to note that this technique shares one important characteristic in common with most other sophisticated pre-execution methods - ultimately, they depend on the prior application of detection (or formal verification) techniques in order to certify an executable file as "clean" in the first place.

Keeping Ken Thompson's admonition "on trusting trust" firmly in mind [5], how should a security administrator proceed when faced with programs so large or complex that "trust, but verify" is not a feasible option? We suggest that - in the middle ground between the two extremes of exhaustively provable correctness and trust based on nothing more substantial than personal familiarity with, or a background security check on, a program's writer - the MCT (acting to assist a human analyst) can provide a practical alternative basis for trust.

\subsection{Simple Scanners and Monitors}

Simple scanners such as McAfee's Scanv or Norstad's Disinfectant are by and large the most common pre-execution method in use today. Typically, the user will invoke a scanner to search the static text of a binary program for fixed patterns (bitstrings) that match those of known malicious programs. If none of those bitstrings are found, the user then proceeds to execute the program. Thus these scanners boast a very good record in defending against known malicious programs, such as polymorphic viruses that use a known "Mutation Engine", but they carnsot be applied in general to finding new malicious code, or even to finding familiar malicious code protected by a "Muta- 
tion Engine" that is, itself, slightly mutated. Another popular approach uses simple monitors to observe program execution and detect potentially malicious behavior at run time. Such monitors usually sit astride the system call interface, e.g., to watch all disk accesses and ensure that no unauthorized writes are performed. Unfortunately such techniques incur a substantial speed penalty during execution of normal programs, and typically become quite a nuisance to the user.

To be effective, these programs must also err on the conservative side, resulting in many false alarms which require user interaction. But in these interactions, current techniques require the user to make relatively immediate (and usually uninformed) decisions regarding whether the program should be allowed to proceed. Such decisions would benefit immensely from the opportunity to explore a trace of the program's history, as well as its then-current execution state.

\subsection{Encryption \& Watchdog Processors}

Encryption is another method of coping with the threat of malicious code. Lapid, Ahituv, and Neumann [7] use encryption to defend against Trojan horses and trapdoors. When correctly implemented, encryption techniques are quite effective against many types of malicious code, but the cost of such a system is high due to the required hardware. Similarly, watchdog processors [8] also require additional hardware. Such processors are capable of detecting invalid reads/writes from/to memory, but they require additional support to effectively combat virises. Also, both of these methods are dependent on the prior existence of a "clean" version of every program that is to be executed. As mentioned, to certify such copies as "clean" in the first place requires either formal verification or a malicious code detection capability, which is the subject of the present paper.

\section{Review of Dynamic Analysis using Events}

Over the last few years, we have developed a powerful, state-of the-art debugger called Dalek [9]. Dalek incorporates two significant advances over traditional debuggers: it features a fully-programmable language for manipulating the debugging environment, and it provides extensive support for user-definable events.

The MCT user's environment was designed in accordance with the philosophy underlying the Dalek debugger, and features analogous to those in Dalek have been incorporated into the MCT. But we have also customized the MOT environment, in light of its specific mission to help ferret out malicious code. We believe that "dynamic analysis" (and the development of appropriate methodologies for it) should be seen as representing an extremely promising avenue of inquiry, rather than as being just a fancy word for the sorts of things people have always done with traditional debuggers.

By fully programmable, we mean the MCT is an extendible environment, in a similar sense that the Emacs text-editor is extendible. But due to the nature of the MCT's mission, these general-purpose language constructs have been fully integrated with traditional application-specific debugging features such as breakpoints and singlestepping.

Like the Dalek debugger, the MCT also provides automated support for detecting hierarchical events - occurrences of interesting activities during the execution of the 
suspicious program. This capability allows the MCT to represent the suspicious program's behavior in terms of whatever higher-level abstractions have been defined by the security analyst.

In some ways, an event is conceptually similar to a tuple in a relational database - once the structure of a particular database table has been defined by the user, every occurrence of an event of that type that is detected by the MCT will have its attributes recorded permanently, as fields in a newly inserted tuple. That is, when the MCT detects an event occurrence, it causes a corresponding tuple (or record) to appear in the appropriate database table. The attributes associated with an event should contain information sufficient to characterize a particular occurrence of that event, allowing it to be distinguished from other instances of the same event. The code written by a security analyst for an event's definition can cause it, upon activation, to assign values to these attributes from variables in the suspicious program, from variables in the "outer" MCT environment, or from computation based on a combination of such variables.

In addition to defining an event as a template for passive data, the security analyst also needs to define an active, procedural aspect for that event. This is accomplished by writing a body of code in the MCT's language, and associating it with that event. The purpose of this code, when activated, is to recognize exactly those conditions in the suspicious program's execution state that the security analyst has specified as constituting a valid occurrence of this particular type of event.

This event-recognition code can be executed manually by the security analyst as s/he single-steps the suspicious program, or it can be executed automatically by the MCT, if the analyst has bound that event's code to a breakpoint, or to a rarige of breakpoint addresses. Events whose code is activated in this manner are called primitive events.

The MCT also supports high-level events. When defining a high-level event, one must specify the names of all lower-level events on which it depends. A high-level event is not explicitly raised; instead, the MCT can automatically trigger a high-level event's code into executing whenever an occurrence of a primitive event on which that highlevel event depends is successfully recognized. The high-level event's code will have access to all the attributes of its lower-level constituent events, as well as access to the "raw" state of the suspicious program and to variables defined in the "outer" MCT environment.

Note that the security analyst can define a high-level event whose recognition may depend on lower-level constituent events whose occurrences are widely separated in time. For a concrete example of a network of events used to detect self-propagating code, see [1].

Viewed from the perspective of a relational database, a high-level event is conceptually akin to an ongoing query: In defining a high-level event, the security analyst poses a query. The MCT then provides incremental answers to that activated query, as the behavior of the suspicious program causes new occurrences of primitive event/attribute tuples automatically to be inserted in the database.

The "execution history database" maintains a record of all recognized event occurrences and their attributes. It may be browsed selectively by the security analyst in interactive mode, or accessed programmatically via access functions written in the MCT's language. 


\section{Architecture of the MCT}

One design gual for the MCT is that it be as universal as possible. That is, the testbed should in principle be capable of analyzing both source code and executable files from different processors and different operating systems. However, to achieve such broad applicability, we would have to develop various front-ends and back-ends for the MCT. In addition, because of the radically different "security architectures" (or lack thereof) on different platforms, that portion of the MCT between the front-ends and back-ends - that portion common to all platforms - could turn out to be the null intersection. Nevertheless, we feel that using a common machine-independent internal form language may illuminate aspects common to many security architectures.

\subsection{Initial Program Loading}

If started, for example, with an "executable" file, a front-end will need to understand any loading (and possibly some dynamic linking) conventions of the target operating system. The front-end will also need to know the processor type of the machine code in order to properly translate it into the Lisp-based internal form. A back-end will need to emulate any dynamic linking operations of the target operating system, as well as its system call interface. Thus, for example, if a program running under the MCT "writes" a file and then "reads" it back again, it should not be apparent to that program that it is not, in fact, running directly on the target CPU and operating system.

Either before or after translation of an executable program into an internal form, the MCT might also search it to identify any known standard system-library routines. Assuming those routines are "clean", this step could significantly reduce the size of the problem. In addition, since the type of every parameter required by a system-library routine is known, this information permits subsequent phases of the analysis to infer the types of any variables in the suspicious program that are passed as arguments to those system-library routines.

\subsection{Program Representation - Internal Form Language}

In order for the MCT user profitably to apply various analysis tools, those tools m"st share a common representation of the suspicious program that is the subject of their analysis.

To analyze the behavior of an executable machine code program, we must first translate its code into our internal form language. We have designed a set of procedures that, given a 2-tuple (Memory_Address, Memory_Contents), will translate its Memory Contents into our internal form language. Because not all assembler instructions have the same length, it behooves us to explicitly represent the original Memory_Address as another field in the internal form representation. This will allow the translation tool easily to access other 2-tuples representing the next few adjacent $\mathrm{Mem}$ ory_Addresses, should the need arise, in order to complete the job of disassembling a single long instruction.

The internal form language was deliberately designed to include only a small number of basic operators, thus simplifying the analysis. These operators are closely related to the hardware operations on a microprocessor, allowing convenient translation from machine code into the internal form. Typical basic operations of the internal form 
language include READ or WRITE to a Memory_Address or Register. As an example of the syntax of the internal form, an indirect write of 0 through register $\mathrm{CX}$ might look like:

( WRITE.BYTE 0, (ADD (READ.WORD CX) \#0x01AE) )

The internal form is a Lisp-like language, whose order of evaluation is the same as that of Lisp. By defining the internal form's operators (e.g., "READ.WORD") as functions in Lisp, a program written in the internal form language can be interpreted by any standard Lisp system. Thus, by using Lisp as the "native language" of the MCT, dynamic analysis can readily simulate the execution of a program that has been translated from machine code into the internal form.

\subsection{Memory Model for the Code Segment}

Although the translator will produce a string of code in our internal form language, the MCT must store much more than just that string of syntax. To adequately represent even a 1-byte instruction of the original machine code, the MCT uses an elaborate data structure that also stores the original (Memory_Address, Memory_Contents) 2-tuple, along with various auxiliary fields to record other information that may be computed by dynamic analysis techniques. Representations of every Memory_Address in a suspicious program's code segment are stored in a table in the MCT.

\subsection{Memory Model for the Data Segment}

Cells in a suspicious program's data memory can be represented by the same structures as are used for its code, although at first it might appear that only the (Memory_Address, Memory_Contents) fields are needed. These representations of its data can be stored in the same table as the representations of its code. Named registers on the target CPU are treated as a special case of data memory. The MCT interpreter "allocates" data memory only as required by the dynamic behavior of the program (i.e., for the run time stack and local variables, and for memory that is explicitly allocated dynamically via calls to malloc). We must also load the MCT interpreter with any initialized data in the original executable machine code file, as well as any sections of DOS we think the program might attempt to access directly (e.g., the interrupt vector table).

\section{Experience Using the Malicious Code Testbed}

The MCT is written in Common Lisp, and its execution of internal form code in a simulated PC/DOS environment on a Unix workstation is several orders of magnitude slower than genuine execution of the original machine code on a PC platform. Nevertheless, because the security analyst can define events, and then leave the MCT to run unassisted for long periods to watch for occurrences of those events, this time penalty is acceptable.

In order to detect self-modifying code, we have included several predefined events in a standard library for the MCT. These events record every memory access - attributes such as the memory access mode (i.e., Read, Write, or eXecute), and the memory address and contents. Thus, we can perform a relational join within this table, e.g., 
if a particular location has been modified by some instruction, we can determine the address of the responsible instruction, and the contents of that instruction, even if they have been modified subsequently. The overhead incurred by this recordkeeping is one reason for the MCT's slow execution.

In implementing the MCT, we are extending the boundaries of our simulated DOS environment incrementally, as necessitated by the demands of our test programs for DOS/BIOS system services. Currently, the DOS system call interface is still somewhat skeletal. Our simulation of the PC hardware is also fairly rudimentary, e.g., we do not currently simulate the periodic clock tick interrupts, and thus we avoid their associated processing time.

As mentioned, the MCT is an extendible, customizable environment. Thus, the exact nature of the "display" it presents to the user is a matter of personal choice. In the sample displays that follow, we utilize a highly verbose mode, that, in most situations, would present the security analyst with far more undigested, low-level information than desired. Nevertheless, on occasion this level of detail is desirable, and is certainly justified in this case on expository grounds.

The Malicious Code Testbed Displays a "Trace" of Program Execution

*** Initializing Low-DOS *** for Compaq 386, DOS 3.31 : 0x0000-0x1000

MCT will simulate $\rightarrow$ JMP $0 \times 1195$ at IP $0 \times 1100::$

$\rightarrow((J U M P(T$ \#x1195)))

MCT will simulate $\rightarrow$ CLD at IP 0x1195::

$\longrightarrow(($ WRITE.F DF \#x0))

MCT will simulate $\rightarrow$ MOV AH, OxE0 at IP 0x1196::

$\longrightarrow(($ WRITE.B AH (REĀD.B (CONST \#xE0))))

MCT will simulate $\rightarrow$ INT Ox21 at IP 0x1198::

$\longrightarrow$ ((LIB INT \#x21))

MCT will simulate $\rightarrow$ CMP AH, OxE0 at IP 0x119A ::

$\longrightarrow(($ WRITE.F AF (AUX (- (READ.B AH) (READ.B (CONST \#xE0)))))

(WRITE.F OF (OVERFLOW (- (READ.B AH) (READ.B (CONST \#xE0)))))

(WRITE.F PF (PARITY (- (READ.B AH) (READ.B (CONST \#xE0)))))

(WRITE.F SF (SIGN (- (READ.B AH) (READ.B (CONST \#xE0)))))

(WRITE.F ZF (ZERO (- (READ.B AH) (READ.B (CONST \#xE0)))))

(WRITE.F CF (CARRY (- (READ.B AH) (READ.B (CONST \#xE0))))))

MCT will simulate $\rightarrow$ JNC \#x11B5 at IP 0x119D ::

$\longrightarrow((\operatorname{JUMP}((=0$ (READ.F $\mathrm{F}))$ \#x11B5) $(\mathrm{T} \# \times 119 \mathrm{~F})))$

MCT will simulate $\rightarrow$ MOV AX, CS at IP 0x11B5::

$\longrightarrow(($ WRITE.W AX (READ.W CS $)))$

MCT will simulate $\rightarrow$ ADD AX, \#x10 at IP 0x11B7 ::

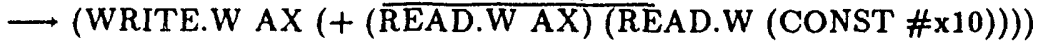


The first example MCT display above provides the conceptual equivalent of a program "trace", such as might be provided by a debugger. For each newly executing instruction, the MCT displays the 8088 assembler mnemonic, the address of that instruction, and its translation into our internal form language (which is then executed by the Lisp interpreter):

In the next example, the security analyst has programmed the MCT to display a more high-level message immediately upon detecting an occurrence of the event named $S E L F-M O D-C O D E$. In this particular case, a memory location that was initially accessed in modes Read, then Write, is subsequently accessed in eXecute mode. The event code, written by the security analyst, notifies him after each subsequent $e X e c u t e$ access, and also provides some higher-level information it has computed - namely, which instruction was responsible for modifying the instruction the MCT just executed.

The Malicious Code Testbed Detects Self-Modifying Code

MCT will simulate $\rightarrow$ JMP-INTER-SEG 0x3FC 0x000 at IP 0x123D :: $\longrightarrow((\mathrm{JUMP}-\mathrm{ABS} \# \times 3 \mathrm{FC} \# \times 0))$

MCT will simulate $\rightarrow \underline{\text { REP }}$ at IP $0 \times 3 F C::$

$\rightarrow(($ PREFIX 'REP \#x1))

SELF-MOD-CODE EVENT - Location 0x3FC modified by address 0x121B

MCT will simulate $\rightarrow$ REP MOVS at IP 0x3FD ::

$\longrightarrow(($ WRITE.W (ES-SHIFT (READ.W DI)) (READ.W (DS-SHIFT (READ.W SI))))

(WRITE.W SI (+ (READ.W SI) \#x2)) (WRITE.W DI (+ (READ.W DI) \#x2)))

SELF-MOD-CODE EVENT - Location 0x3FD modified by address 0x121B

MCT will simulate $\rightarrow$ REP MOVS at IP 0x3FD ::

$\longrightarrow(($ WRITE.W (ES-SHIFT (READ.W DI)) (READ.W (DS-SHIFT (READ.W SI))))

(WRITE.W SI (+ (READ.W SI) \#×2)) (WRITE.W DI (+ (READ.W DI) \#x2))

SELF-MOD-CODE EVENT - Location 0x3FD modified by address $0 \times 121 \mathrm{~B}$

In the case above, the MOVS instruction that was modified is being repeated because of its prefix, REP. Thus, every time it repeats, the MCT displays the fact that it detected another occurrence of the event, SELF-MOD-CODE. The security analyst might decide to redefine this event so that when not in "verbose" mode (as determined by examining a variable in the "outer" MCT environment), it will quietly record all eXecute accesses to this location after the first Read, Write, eXecutc sequence, but will not announce those subsequent occurrences of the event immediately. 
In the next example, after a section of malicious code has decrypted itself (not shown), the decrypted code proceeds to read the realtime clock twice in rapid succession to check whether it is being single-stepped under a debugger. If less than 1 second has elapsed, it assumes it is not being watched, and then attempts a reboot.

Decrypted Code Reads Clock to Check if being Single-Stepped under Debugger;

If Not Being Watched, It Then Attempts a Reboot

MCT will simulate $\rightarrow$ MOV at IP $0 \times 1035:$ :

$\longrightarrow$ ((WRITE.B AH (READ.B (CONST 0x2))))

SELF-MOD-CODE EVENT - Location 0x1035 modified by address 0x105C

MCT will simulate $\rightarrow$ INT at IP $0 \times 1037::$

$\longrightarrow(($ LIB INT 0x1A))

Program desires to read 24-hr realtime clock (base 10):

Enter hours: 11

Enter minutes: 15

Enter seconds: 03

Enter hundredths: $\quad 00$

SELF-MOD-CODE EVENT - Location 0x1037 modified by address 0x105C

MCT will simulate $\rightarrow$ PUSH at IP $0 \times 1039::$

$\longrightarrow$ ((WRITE.W SP (- (READ.W SP) 0x2))

(WRITE.W (SS-SHIFT (READ.W SP)) (READ.W DX)))

SELF-MOD-CODE EVENT - Location 0x1039 modified by address 0x105C

MCT will simulate $\rightarrow$ MOV at IP 0x103A ::

$\longrightarrow$ ((WRITE.B AH (READ.B (CONST 0x2))))

SELF-MOD-CODE EVENT - Location 0x103A modified by address 0x105C

MCT will simulate $\rightarrow$ IN'T at IP $0 \times 103 \mathrm{C}::$

$\longrightarrow(($ LIB INT 0x1A))

Program desires to read 24-hr realtime clock (base 10):

Enter hours:

11

Enter minutes: 15

Enter seconds: 03

Enter hundredths: $\quad 00$

SELF-MOD-CODE EVENT - Location 0x103C modified by a.ddress 0x105C

MCT will simulate $\rightarrow$ POP at IP 0x103E ::

$\longrightarrow$ ((WRITE.W AX (READ.W (SS-SHIFT (READ.W SP))))

(WRITE.W SP (+ (READ.W SP) 0x2)))

SELF-MOD-CODE EVENT - Location 0x103E modified by address 0x105C 
MCT will simulate $\rightarrow$ CMP at IP 0x103F :

$\rightarrow(($ WRITE.F AF (AUX (- (READ.B DH) (READ.B AH))))

(WRITE.F CF (CARRY (- (READ.B DH) (READ.B AH))))

(WRITE.F OF (OVERFLOW (- (READ.B DH) (READ.B AH))))

(WRITE.F PF (PARITY (- (READ.B DH) (READ.B AH))))

(WRITE.F SF (SIGN (- (READ.B DH) (READ.B AH))))

(WRITE.F ZF (ZERO (- (READ.B DH) (READ.B AH)))))

SELF-MOD-CODE EVENT - Location 0x103F modified by address 0x105C

MCT will simulate $\rightarrow$ JNZ at IP 0x1041 :

((JUMP ((=0 (READ.F ZF)) 0x1063) (T 0x1043)))

SELF.MOD.CODE EVENT - Lucation 0x1041 modified by address 0x105C

MCT will simulate $\rightarrow \underline{\text { AND }}$ at IP $0 \times 1043::$

$\longrightarrow$ ((WRITE.F AF UNDEF) (WRITE.F CF 0x0) (WRITE.F OF 0x0)

(WRITE.B CL (LOGAND (READ.B CL) (READ.B (CONST 0x1))))

(WRITE.F PF (PARITY (READ.B CL))) (WRITE.F SF (SIGN (READ.B CL)))

(WRITE.F ZF (ZERO (READ.B CL))))

SELF-MOD.CODE EVENT - Location $0 \times 1043$ modified by address 0x105C

MCT will simulate $\rightarrow$ CMP at IP 0x1046::

$\longrightarrow$ ((WRITE.F AF (AUX (- (READ.B CL) (READ.B (CONST 0x1)))))

(WRITE.F CF (CARRY (- (READ.B CL) (READ.B (CONST 0x1)))))

(WRITE.F OF (OVERFLOW (- (READ.B CL) (READ.B (CONST OX1)))))

(WRITE.F PF (PARÏTY (- (READ.B CL) (READ.B (CONST 0x1)))))

(WRITE.F SF (SIGN (- (READ.B CL) (READ.B (CONST 0x1)))))

(WRITE.F ZF (ZERO (- (READ.B CL) (READ.B (CONST Ox1))))))

$S E L F-M O D-C O D E E V E N T$ - Location $0 \times 1046$ modified by address 0x105C

MCT will simulate $\rightarrow$ JNZ at IP $0 \times 1049::$

$\rightarrow((J U M P((=0$ (READ.F ZF)) 0x1063) (T 0x104B)))

SELF-MOD.CODE EVENT - Location 0x1049 modified by address 0x105C

MCT will simulate $\rightarrow$ INT at IP $0 \times 104 B$ ::

$\rightarrow(($ LIB IN'T 0x19))

Program attempting a Reboot - OK to proceed? N

$S E L F-M O D$-CODE EVENT - Location 0x104B modified by address 0x105C

Decrypted Code Reads Clock to Check if being Single-Stepped under Debugger;

If Not Being Watched, It Then Attempts a Reboot 


\section{Future Research}

One direction to pursue is to focus on the so-called "polymorphic" viruses that, by using unknown "Mutation Engines", can easily evade static scanners. Our event-based dynamic analysis techniques should be able to handle all polymorphic "mutants", since all polymorphic variants of a given virus should share a common behavioral profile a common dynamic canonical form.

We also plan to return to implementing one of our original design goals for the MCT, namely integrating static analysis tools [1] into the common MCT environment. We feel there is great potential for the complementary use of these two families of analysis techniques, leading ultimately to the development of a more rigorous detection methodolugy.

It may be that a two-tier detection scheme will be warranted - an efficient-running coarse-grain event filter that could quickly screen large sections of code, and a slower, more thorough, fine-grain event mesh for especially critical systems or software.

\section{Acknowledgements}

We thank Doug Mansur for his valuable insights.

\section{References}

[1] R. Crawford, R. Lo, J. Crossley, G. Fink, P. Kerchen, W. Ho, K. Levitt, R. Olsson, M. Archer. "A Testbed for Malicious Code Detection: A Synthesis of Static and Dynamic Analysis Techniques ", Secure Networks - Proceedings: Fifth International Computer Virus \& Security Conference, March 1992, pp. 225-236.

[2] F. Cohen. "Computer Viruses - Theory and Experimen is", Computers \& Security, Vol. 6, 1987, pp. 22-35.

[3] Len Adleman. "An Abstract Theory of Computer Viruses" (abstract), CRYPTO '88.

[4] M. Harrison, W. Ruzzo, and J. Ullman. "Protection in Operating Systems", CACM, Vol. 19, No. 8, Aug. 1976, pp. 461-471.

[5] K. Thompson. "Reflections on Trusting Trust", Comm. ACM, Vol. 27, No. 8, 1984, pp. 761-763.

[6] F. Cohen. "A Cryptographic Checksum for Integrity Protection", Computers $\mathcal{G}$ Security, Vol. 6, 1987, pp. 505-510.

[7] Y. Lapid, N. Ahituv, and S. Neumann. "Approaches to Handling 'Trojan Horse' Threats", Computers \& Security, Vol. 5, 1986, pp. 251-256.

[8] A. Mahmood and E. J. McCluskey. "Concurrent Error Detection Using Watchdog Processors-A Survey", IEEE Transactions on Computers, Vol. 37, No. 2, 1988, pp. 160-174.

[9] R. Olsson, R. Crawford, and W. Ho. "A Dataflow Approach to Event-based Debugging", SOFTWARE-Practice and Experience, Vol. 21, No. 2, Feb. 1991, pp. 209-229. 

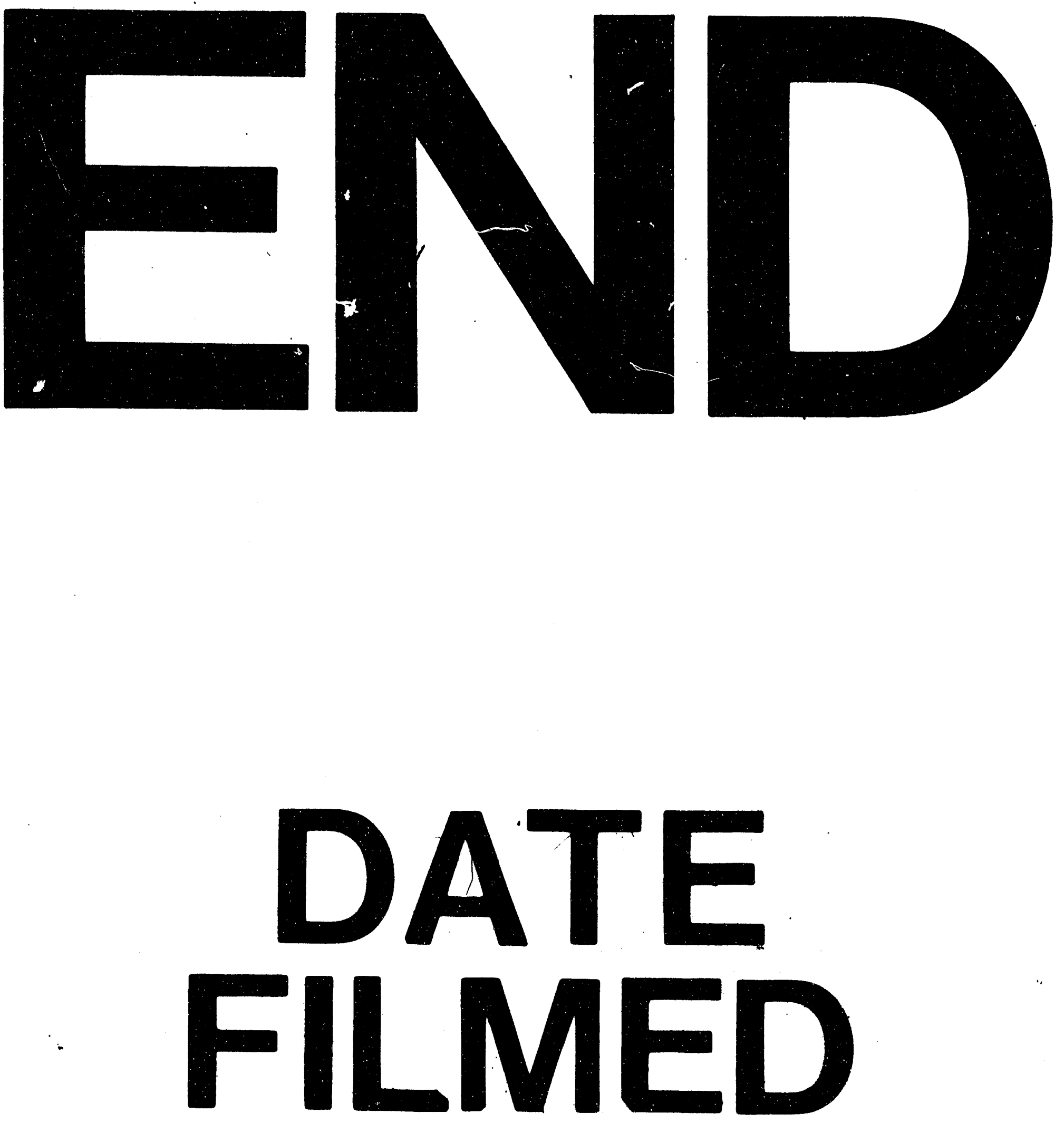

1

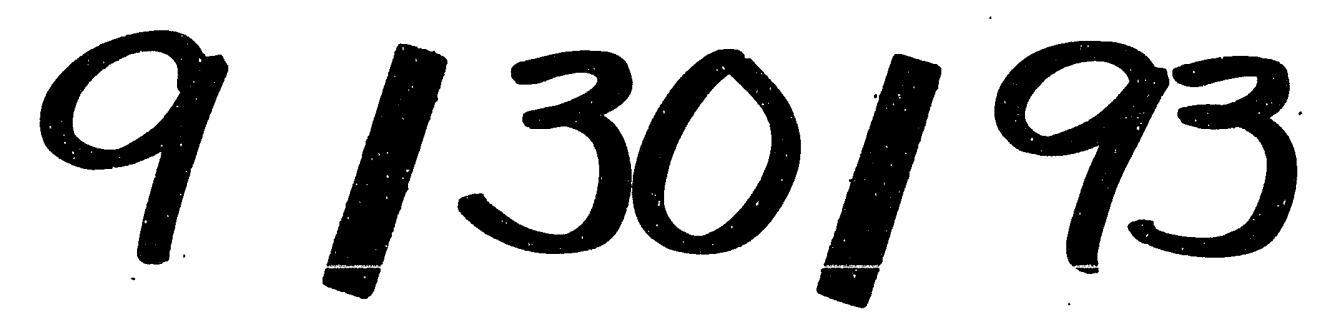


